\title{
Test yourself: painless hand mass
}

\author{
Sarah Fenerty $^{1}$ (D) $\cdot$ Stephen Ling $^{1} \cdot$ Congli Wang $^{2} \cdot$ Omer Awan $^{1} \cdot$ Sayed Ali $^{1}$
}

Published online: 29 November 2016

(C) ISS 2016

HISTORY: A 34 year-old man presented to his primary care physician with a painless mass along the dorsum of the second metacarpal neck of 8-9 months duration. There was no history of trauma. (Figs. 1, 2 and 3)

The diagnosis can be found at doi: 10.1007/s00256-016-2544-y

Sarah Fenerty

sarah.fenerty@tuhs.temple.edu

Stephen Ling

stephen.ling@tuhs.temple.edu

Congli Wang

congli.wang@tuhs.temple.edu

Omer Awan

omer.awan@tuhs.temple.edu

Sayed Ali

alisayan@tuhs.temple.edu

1 Department of Radiology, Temple University Hospital, 3401 North Broad St., Philadelphia, PA 19140, USA

2 Department of Pathology, Temple University Hospital, 3401 North Broad St., Philadelphia, PA 19140, USA

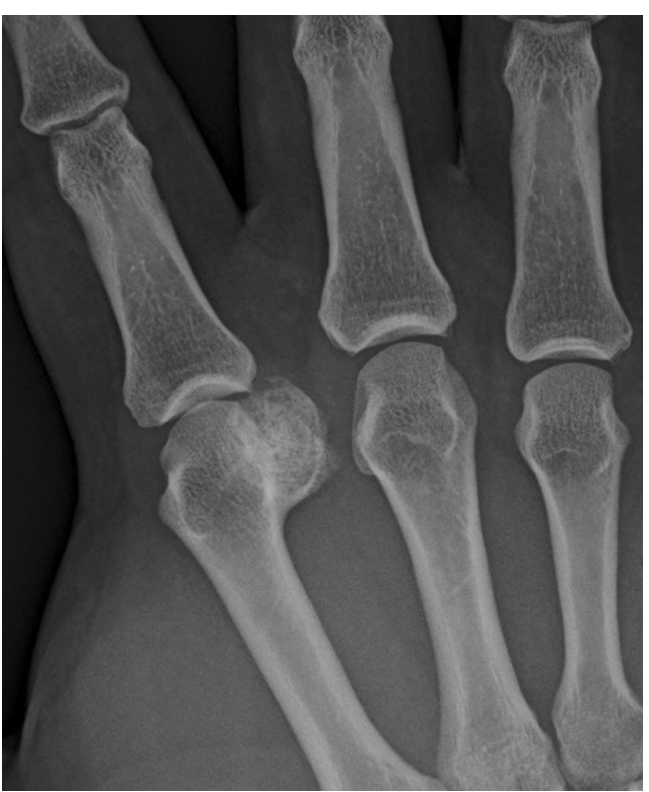

Fig. 1 AP radiograph of the right 2nd-4th metacarpals and proximal phalanges 
Fig. 2 Axial (a), coronal (b), and sagittal (c) noncontrast $\mathrm{CT}$ of the right hand at bone windows
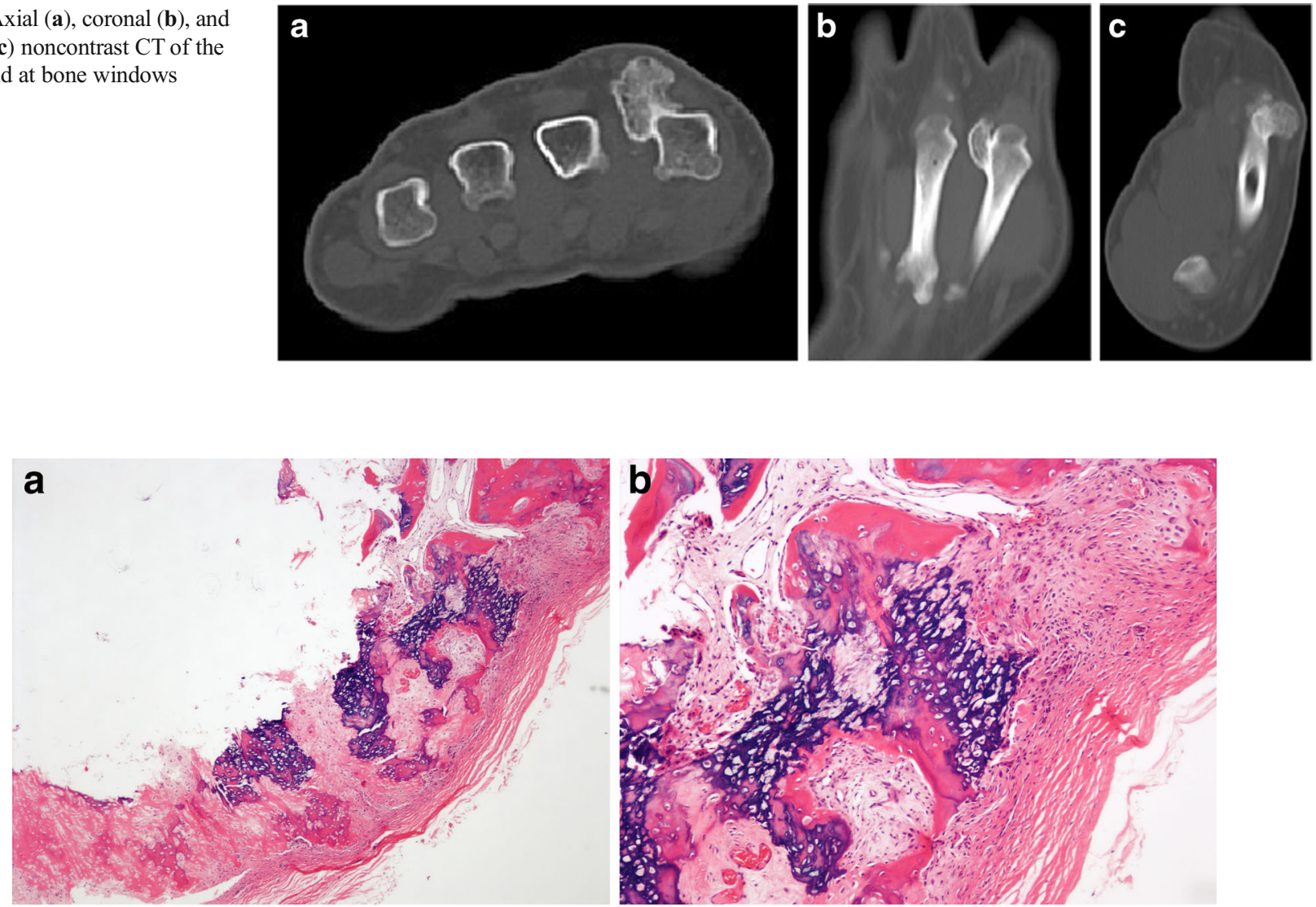

Fig. 3 Histopathological photomicrographs (hematoxylin and eosin staining) at (a) 40x power and (b) 100x power

\section{Compliance with ethical standards}

Funding information No funding was received for this article. 